\title{
La Educación Geográfica y la Formación Ciudadana: Conceptualización, vinculación y puesta en práctica
}

\author{
Annie Vargas Hernández*
}

\section{Resumen}

Este artículo consiste en una revisión bibliográfica que busca analizar los diferentes conceptos que se han planteado alrededor de las disciplinas de la educación geográfica y la formación ciudadana, por medio de, por un lado, las comparaciones entre las diferentes concepciones y, por otro lado, buscando la vinculación entre ellas. Con esto, el objetivo es aclarar el objeto de estudio de la educación geográfica y la formación ciudadana, en el que se pueda encontrar el punto de convergencia entre ambas, así como ejemplificar la puesta en práctica de su enseñanza.

\section{Palabras clave}

educación geográfica, formación ciudadana, territorio, ciudadano.

\begin{abstract}
This is a bibliographical research that intents to analyze the different concepts towards the disciplines of geographic education and education for citizenship, based on comparisons among the different conceptions, and looking for the relation between them. The objective is to clarify the under study of geographic education and education for citizenship, y find the focal point, as well as the implementation of the teaching process.
\end{abstract}

\section{Keywords}

geographical education, education for citizenship, territory, citizien. 


\section{Introducción}

Este artículo intenta exponer las diferentes concepciones que se han desarrollado en torno a los términos de la educación geográfica y de la formación ciudadana. El desarrollo, se estructura en tres partes: revisión de conceptos, vinculación entre ambos y la puesta en práctica de estas disciplinas en tres países Latinoamericanos.

En la primera parte, se presentan las distintas definiciones de educación geográfica así como de formación ciudadana desde diversas perspectivas, en las que se rescatan los principales elementos de cada una de estas disciplinas y se comparan entre sí, lo que permite sintetizar e identificar el objetivo primordial de cada concepción. Para el segundo apartado, se intenta señalar los puntos de convergencia entre la educación geográfica y la formación ciudadana, el cómo la primera es complemento de la segunda y viceversa. Se toma como base los puntos identificados tanto por el Dr. Fabián Araya Palacios como por otros autores.

Para el análisis de la puesta en práctica de la educación geográfica y la formación ciudadana en la 2 enseñanza, se toma como referencia estudios realizados en Chile, Colombia y en Costa Rica. Posteriormente, se expondrán las conclusiones alcanzadas producto de la revisión bibliográfica, del análisis y discusión de los conceptos, así como de la puesta en práctica de ambas disciplinas en diversos contextos latinoamericanos.

\section{Conceptualización: Educación Geográfica y Formación Ciudadana}

En este apartado se definen los conceptos de la educación geográfica y el de formación ciudadana. Se expone en qué consiste cada una, con el fin de comprender su objeto de estudio. 


\subsection{Educación Geográfica}

Para comprender la educación geográfica, primero se deben conocer las diferentes definiciones que se han establecido alrededor de esta disciplina y encontrar el punto en común entre todas con el fin de esclarecer y comprender mejor su objetivo en la enseñanza.

Se hace oportuno empezar por la definición que desarrolla Souto (2004), la cual expone a "la geografía escolar como un conjunto de conocimientos que son útiles para la socialización de los alumnos, pues les permiten interpretar sus identidades personales y colectivas". (p.13) Este, enfatiza en que la educación geográfica es un instrumento que le permite al estudiante socializar gracias al conocimiento que brinda su enseñanza.

Además, se dice que "educar geográficamente será formar un individuo capaz de comprender el lugar que ocupa en el mundo y las relaciones particulares que establece con los demás y con su entorno, ya sea local, regional y/o global". (Buitrago, 2005, p. 1) Esta descripción resulta muy interesante, ya que, no sólo hace referencia a la socialización del individuo a través de las relaciones en las que pueda implicarse, sino que también hace mención a que este debe estar consciente del lugar que ocupa, así como del entorno donde se encuentra. Ya no se habla del territorio como un solo conjunto, puesto que mencionan las diferentes escalas a las que se puede expandir el análisis territorial.

Otros autores, como Claudino y Spinelli(2012), dicen que "la Geografía nos ayuda a buscar el mundo, a comprenderlo - pero, más que su interpretación, la educación geográfica debe significar la intervención activa en la resolución de los problemas territoriales, a diferentes escalas". (p. 57) La concepción anterior, contribuye a las definiciones preliminares en un nuevo aspecto, puesto que se señala al individuo como el ente principal en la resolución de los problemas que se desarrollen en el territorio.

Para De la Calle (2013)

la enseñanza de la Geografía debería contribuir a la formación de ciudadanos que realicen una mirada crítica a la realidad que nos rodea. La enseñanza de la Geografía debe contribuir al desarrollo de competencias, poniendo la vista hacia el objetivo de qué tipo de ciudadanos se pretende formar en el sistema educativo actual. (p. 123) 
En este punto, se observa la educación geográfica como una vía óptima que permite la formación y el desarrollo de ciudadanos capaces de comprender la realidad en la que viven. Igualmente, la educación geográfica para Araya (2014) se define como el "área del conocimiento educativo que integra el saber pedagógico y el conocimiento geográfico”. (p.7) Esta rama de la educación busca la relación del ser humano con los acontecimientos que se desarrollan en el ámbito social, geográfico y ambiental, tomando en cuenta la variable espacial dentro de esta dinámica.

La educación geográfica pretende la integración de la pedagogía con el conocimiento y reconocimiento del espacio geográfico, es decir, el lugar en el que el ser humano se desenvuelve. Busca que la persona tenga conocimiento del espacio geográfico en el que crece y se desarrolla como ciudadano. Es por ello que, la educación geográfica ha adquirido un rol importante dentro de la enseñanza.

Aunado a lo anterior, Pulgarín (2011) propone que la geografía sea una disciplina integradora dentro del área de la enseñanza, ya que, esta "permea encuentros conceptuales y de contenido, que permiten el estudio comprensivo del mundo real; que puede asumirse desde una de las acepciones de espacio geográfico, por ejemplo la de territorio". (p. 2-3)

Para el ciudadano es más sencillo contextualizar su desenvolvimiento desde el concepto de territorio que desde el espacio geográfico, ya que, este último puede tornarse un poco ambiguo y confuso. Sin embargo, es importante recordar que el espacio geográfico es el "espacio socialmente construido" (Santos 2000, citado en Pulgarín, 2011, p. 8), y es allí, donde se manifiestan todos los procesos que involucran a los diferentes actores sociales, a través del tiempo. Por lo que, se realza la importancia de que los ciudadanos tomen conciencia del territorio en el que habitan, y de todos aquellos procesos que se desarrollan en él. El estudio del territorio, como parte de la educación geográfica en las ciencias sociales permite "la formación de un ciudadano ubicado, capaz de hacerse visible en un lugar, de otorgar sentido al territorio que habita y a mostrar compromiso en su transformación”. (Pulgarín, 2011, p. 11) Esta visión concuerda con lo que expresan Arenas y Salinas (2013), cuando fundamentan que

El posicionamiento de la Geografía como disciplina científica, capaz de generar conocimiento útil para la sociedad, tiene directa relación con la alfabetización científica de los habitantes de un país o región. De esta forma, unas personas capaces de comprender los fenómenos desde un punto de 
vista geográfico contribuirán a una mejor apropiación de las problemáticas del territorio vivido, es decir, alfabetizados geográficamente, siendo este un propósito fundamental de la propia Educación Geográfica. (p. 144)

Asimismo, la educación geográfica, según Rodríguez (2012), tiene como meta, la cual consiste en ayudar a los estudiantes a pensar el espacio desde una perspectiva reticular; es decir, visualizando el territorio como el lugar donde se dan las relaciones de convivencia, conflicto, dominio y cooperación.

Una vez expuestas todas las definiciones, se puede concluir que la educación geográfica pretende formar al ciudadano para que reconozca el territorio en el que habita para que tenga conciencia de ese espacio, y de las diferentes escalas en las que puede ser comprendido, que entienda todas las relaciones y particularidades que se desarrollan en dicho territorio, y que a su vez, posea la capacidad de resolver los conflictos que ahí se generen.

Es así como, se logra identificar que el objeto de estudio de la educación geográfica es un conocimiento estratégico indispensable para el ciudadano, puesto que el conocimiento espacial de los territorios "permite formar personas inteligentes y capaces de comprender los sistemas espaciales y ambientales de los cuales forman parte". (Gallagher 2012, citado en Araya, 2014, p. 8)

\subsection{Formación Ciudadana}

La formación ciudadana es según Araya, Arenas, Bruno, Figueroa, Salinas, y Canihuante (2013) una “institucionalidad política y ampliación temática a problemas actuales de la sociedad y a las competencias para resolver conflictos” (p. 37), que además debe estar presente durante la secuencia escolar, y estar orientada para la adquisición de conocimientos y habilidades para relacionarse de forma participativa y democrática en diferentes ambientes. Esta definición da a entender que la formación ciudadana debe dar las herramientas a los futuros ciudadanos para resolver los conflictos que se presenten en la sociedad, además de brindarle el conocimiento y las destrezas para que se involucren de manera participativa y democrática en la sociedad. 
Asimismo, se dice que "la educación para la ciudadanía concierne al conjunto de prácticas y actividades diseñadas para ayudar a todas las personas, niños, jóvenes y adultos, a participar activamente en la vida democrática y practicando sus derechos y responsabilidades" (Olveira, Rodríguez y Touriñan 2006, citado en Medir y Magin, 2012, p. 44). Igualmente, se le da un rol participativo y activo al ciudadano dentro de la definición brindada por estos autores, en la que a su vez, se hace mención de los derechos y responsabilidades que cada uno tiene dentro de la sociedad.

Pagés, citado en Flores (2012), define que el objetivo de la formación ciudadana consiste en "formar ciudadanos y ciudadanas capaces de saber convivir democráticamente con los demás, de participar en la vida social, laboral, cultural y política de su mundo, intentando mejorarla". (p. 80) Hace mención a la convivencia pacífica y democrática que debe existir entre los ciudadanos y de cómo éstos deben participar activamente de la vida en sociedad. La formación ciudadana debe preparar al individuo para incursionarse en la sociedad poniendo en práctica esas habilidades. Noción que coincide con la siguiente, en la que establece que el objetivo de la formación ciudadana "debe ser capacitar a los alumnos para intervenir, participar y convivir como ciudadanos en un mundo plural y diverso como es el actual”. (Gomariz, Gómez, Miralles, y Ortuño, 2012, p. 89)

Gutiérrez (2010), sin embargo agrega que,

La formación ciudadana, ante todo, como proceso, en virtud a su nexo con la pedagogía como fundamento de las ciencias sociales, vinculada al carácter de proceso social de la educación, en relación directa con la sociología y la filosofía de la educación, con la psicopedagogía y la didáctica; proceso educativo que acontece no sólo, pero sí preeminentemente, en la escuela y, muy en particular, en los niveles básicos, orientada por la teoría de los procesos conscientes, para conocer, ser, actuar y transformar (p. 8).

Es decir, la formación ciudadana es una herramienta pedagógica que vinculada al proceso social con la enseñanza como tal, dota al ciudadano de capacidades para desenvolverse y transformarse en la sociedad. Además, se dice que la formación ciudadana es el "proceso pedagógico soportado en la teoría de los procesos conscientes, mediante el cual se forma al ciudadano territorial apoyado en las categorías de la instrucción, la educación y el desarrollo, en contexto, para que ejerza su ciudadanía territorializada". (Gutiérrez y Pulgarín, 2009, p. 44) 
Se define al ciudadano como un ciudadano territorial que se ubica en un lugar determinado y delimitado, que posee la potestad de "ejercer la ciudadanía requerida para construir el anhelo democrático" (Gutiérrez y Pulgarín, 2009, p. 39), mediante su participación activa, social, participativa y de cualquier rol dentro del espacio geográfico en el que se encuentra. Precisamente, esta concepción integra todos los puntos mencionados por los autores anteriores; y además, se agrega un plus que es el territorio y la conciencia que se deben enseñar al ciudadano acerca del lugar donde habita. Se da como objetivo la formación de ciudadanos conscientes que se involucren en la producción social del espacio, ya que, "no se nace ciudadano; se hace, se forma”. (Gutiérrez y Pulgarín, 2009, p. 43)

De esta manera, se puede expresar que la formación ciudadana es aquella disciplina que se encarga de constituir ciudadanos capaces de participar activa y democráticamente dentro de la sociedad, y que además, sean conscientes del territorio que ocupan y tengan la capacidad de resolver los conflictos que se presenten.

\section{Vinculación entre la Educación Geográfica y la Formación Ciudadana}

Una vez expuestas las múltiples definiciones de lo que se entiende por educación geográfica y formación ciudadana, se procede a establecer y señalar la relación existente, así como los puntos de convergencia entre ambas disciplinas. Araya et al (2013), identifican cinco aspectos que vinculan a la educación geográfica con la formación ciudadana, los cuales se tomarán como punto de partida para desarrollar este apartado.

Uno de los puntos de convergencia que estos autores señalan entre ambas disciplinas es la visión multidimensional; ya que, tanto la educación geográfica como la formación ciudadana buscan dar respuesta a una situación a través de la identificación de posibles causas. Esta concordancia tiene relación con lo que apunta Pagés, citado en Araya (2013), al manifestar que, la importancia que tiene la educación geográfica dentro de la formación ciudadana radica en constituir ciudadanos con múltiples capacidades para afrontar diversas situaciones. Es decir, la educación geográfica le da un valor agregado 
a la formación ciudadana, al contribuir con la multicausalidad con el objetivo de resolver conflictos y circunstancias que se presenten en la sociedad, así como en el territorio en el transcurso de lo cotidiano.

Araya et al (2013), establecen que tanto la educación geográfica como la formación ciudadana coinciden en visualizar el entorno local como un recurso de aprendizaje, en el que los futuros ciudadanos perciben y contextualizan lo que pasa a su alrededor a diferentes escalas. Este punto coincide con la siguiente concepción que establece que "las contribuciones de la geografía escolar a la formación de una conciencia espacial ciudadana están relacionadas al significado político cultural del trabajo pedagógico, comprometidas con la realidad socio espacial, desde sus dimensiones locales a las más globales". (Claudino y Spinelli, 2012, p. 56)

Esta conceptualización no sólo hace mención al conocimiento espacial o territorial que debe adquirir el ciudadano en su formación, sino que también menciona la importancia que tiene la concepción de las dimensiones del territorio que habita, debe conocer lo que pasa a nivel global y su impacto a una escala más local. Se adecua bastante a lo expresado por Gutiérrez (2010), al referir que "el valor del individuo que nace depende del lugar que habita". Y que el ciudadano territorial, como él mismo lo define, en ejercicio de su ciudadanía está ubicado en un lugar, en un espacio geográfico delimitado.

Como tercer aspecto de vinculación, se señala la necesidad de que la sociedad responda a ambas disciplinas, es decir, "para ambos cuerpos de conocimiento, la Globalización se constituye como una coyuntura que impulsa a su actualización y revaloración social". (Araya et al, 2013, p. 38) Es importante que el ciudadano tenga la capacidad de adecuarse a los cambios que la sociedad experimenta en su cotidianeidad, especialmente en un mundo que cada vez se encuentra más interconectado. Esto se respalda con lo establecido por Souto (2011), quien enfatiza en que "es posible desarrollar valores fundamentales de la ciudadanía en su ámbito espacial atendiendo a los hechos cotidianos" (p. 40), con una formación ciudadana que parta desde la geografía. El desenvolvimiento del ciudadano en un espacio geográfico (territorio) tomando conciencia de lo que acontece a su alrededor, lo cual le permitirá forjar los principios básicos que se requieren para vivir en sociedad. 
Araya et al (2013) indican que tanto la educación geográfica como la formación ciudadana se compenetran en cuanto a la incursión de estas temáticas al currículo de enseñanza, lo que ellos denominan como el estado de desarrollo. Es decir, se dificulta impartir Geografía, Estudios Sociales e Historia, pues dichas disciplinas deben compartir el tiempo de enseñanza y ejercicio, lo que genera una problemática para que se dé una adecuada gestión curricular. Este punto es un reto importante en la enseñanza de estos componentes, ya que éstas adquieren un papel transcendental en la vida de los ciudadanos, en la que "la enseñanza de la geografía en perspectiva de formación ciudadana se enmarca en un paradigma pedagógico que entiende la enseñanza como proceso de liberación”. (Hierro, G. 1980, citado en Caro, 2012, p. 18)

Al impartir educación geográfica y formación ciudadana, se educa para que los individuos tengan conciencia de sí mismos, autonomía, que adquieran derecho y deberes, y que respondan de manera responsable ante la sociedad. Además de ser partícipes y resolver conflictos en el territorio que habitan. Es por ello que, encontrar un punto de equilibrio dentro del currículo para su instrucción es un desafío que ambas disciplinas buscan solucionar.

Como última relación existente entre la educación geográfica y la formación ciudadana señalada por Araya et al (2013), se encuentra "la forma en que estructuran el aprendizaje a nivel educacional". (p. 38) Ambas buscan fomentar las habilidades y conocimientos en el futuro ciudadano desde la perspectiva de cada disciplina. Esto lo explica de manera expedita y concisa Caro (2012), al expresar que "afrontar la formación ciudadana implica necesariamente la pertenencia a un conjunto de valores tales como, la tolerancia, la responsabilidad de los actos, la búsqueda del bien público. Desde la educación geográfica se pueden desarrollar estos valores fundamentales en su ámbito espacial, atendiendo a los hechos cotidianos, se debe buscar el desarrollo de competencias geográficas que sean útiles a la formación ciudadana". (p.35)

Tanto la formación ciudadana como la educación geográfica dotan de herramientas a los individuos para que ejerzan sus derechos, cumplan con sus deberes, participen democráticamente dentro de la sociedad y resuelvan los problemas del día a día; todo esto desde el territorio que habitan a diversas escalas. El 
educar y formar "ciudadanos geográficamente alfabetizados" (Araya, 2014, p. 8) es el propósito que busca desarrollar la educación geográfica desde la perspectiva de la formación ciudadana.

\section{Puesta en práctica de las dos disciplinas en Chile, Colombia y Costa Rica}

En este apartado, se expondrán diferentes ejemplos de cómo se ha trabajado la educación geográfica y la formación ciudadana en países como Chile y Colombia. Además, se ejemplificará como en Costa Rica se ha incursionado en este tema desde el currículo del Bachillerato de Estudios Sociales y Educación Cívica.

\subsection{Chile}

En Chile se han realizado diversas investigaciones que enfatizan la importancia sobre la vinculación

existente entre educación geográfica y formación ciudadana. Uno de los principales referentes en el desarrollo de este artículo, Fabián Araya Palacios (2013), es de los autores que más han trabajo sobre el tema en ese país. En su estudio titulado Formación Ciudadana desde la Educación Geográfica, Palacios (2013) pone de manifiesto la importancia que supone el desarrollo en conjunto de ambas disciplinas desde la etapa escolar, con el objetivo de formar ciudadanos con conciencia espacial. Además, se muestran ejemplos de cómo se puede trabajar tanto la educación geográfica como la formación ciudadana desde temas que sean de interés para la sociedad, que estén acordes con la realidad que se vive y que permita forjar ciudadanos que contribuyan en la solución de los posibles problemas que afecten a la sociedad.

Por ejemplo, en este escrito se muestra el trabajo realizado por Ivannia Olivares y Juan Rojas (2013), en el que desarrollan la Guía didáctica para la formación ciudadana: Prevención de riesgos naturales en Chile. Enfatiza en que el estudiantado de sexto grado se debe tener conocimientos previos y técnicos sobre el tema de la prevención de riesgos naturales, y que una vez adquiridos es indispensable que el estudiantado tome el conocimiento alcanzado y lo traslade al contexto físico donde habita y logre 
identificar los riesgos de su comunidad. Además, se hace referencia a la importancia del uso de las tecnologías de la información y comunicación (TICs) para el desarrollo de esta guía. (Araya, 2013, p. 148)

Con lo expuesto anteriormente, se evidencia como un tema de interés para la sociedad actual puede ser abordada por estudiantes de sexto año, en el que se fomenta su formación ciudadana y su conciencia sobre el territorio que habita. Es un ejemplo bastante claro de cómo se complementan efectivamente estas dos concepciones.

Otro de los estudios realizados por Araya (2014) es el que se denomina Relevancia de la Educación Geográfica en Chile. Dicho documento menciona varios aspectos de interés como la creación de la Sociedad Chilena de Ciencias Geográficas (SOCHIGEO) con el objetivo de manifestar la relevancia y los intereses que tiene la educación geográfica para el currículo en Chile. Asimismo, se presenta el proceso por el que pasa Chile entre el 2010 y el 2014, en el que se hacen ajustes en el currículo, teniendo como consecuencia una abrumadora, la disminución de los objetivos y contenidos de la educación geografía. La SOCHIGEO y demás interesados en la materia manifiestan la importancia de la educación geográfica y logran que el Ministerio de Educación de este país se replantee dichos ajustes. Un caso similar se presentó en Costa Rica, pero se explicará más adelante.

\subsection{Colombia}

En lo que respecta a Colombia, María Raquel Pulgarín Silva y Alberto León Gutiérrez Tamayo (2009) han realizado varias investigaciones relacionadas con la formación ciudadana y su vínculo con la educación geográfica. Gutiérrez (2010) basa su artículo denominado El ciudadano territorial: propósito de la formación ciudadana en dos investigaciones realizadas en Colombia, que ponen de manifiesto la relevancia que tiene la educación geográfica para la formación del ciudadano. En dicho documento, se cita que el principal objetivo "es contribuir a la educación geográfica colombiana, haciendo visibles y argumentando las posibilidades que otorga la formación ciudadana basada en la teoría de los procesos conscientes, en perspectiva de los estudios del territorio soportados en el enfoque de la geografía crítica, 
para constituir el ciudadano territorial que ha de potenciar la democracia, desde el ejercicio de su ciudadanía”. (Gutiérrez, 2010, p.2)

En la anterior cita, Gutiérrez (2010), se hace referencia al ciudadano territorial que se ha definido, y que según el autor debe ser forjado desde las aulas, se deben dar los insumos a los futuros ciudadanos para que desarrollen las capacidades de análisis y concientización del territorio. Por su parte, Pulgarín (2011) desarrolla en su artículo Los estudios del territorio y su intencionalidad pedagógica, una de las investigaciones también utilizadas por Gutiérrez denominada El estudio del territorio como estrategia para el fortalecimiento de las competencias ciudadanas. Análisis del Programa Urbano Integral de la zona Nor-oriental (PUI-NOR), Medellín-Colombia.

Pulgarín (2011) puntualiza sobre la importancia que se tiene para "avanzar en la formulación e implementación de los estudios del territorio como propuesta pedagógica que flexibiliza el currículo y propende por la educación geográfica". (p. 1) El objetivo, en el que se enmarca el proyecto de investigación que toma tanto Gutiérrez como Pulgarín, muestra la relevancia sobre la integración de la educación geográfica y la formación ciudadana, y de cómo ella es importante en el currículo colombiano.

En el ensayo titulado El espacio geográfico como objeto de enseñanza en el área de las Ciencias Sociales, Pulgarín (2011) menciona que la Ley de General de Educación de 1994 generó un Plan de Estudios con nueve áreas de conocimiento, entre las que rescata las ciencias sociales. Señala la importancia que tiene la integración de esas nueve áreas entre sí dentro del currículo colombiano, y cómo todas ellas son necesarias dentro de la formación del ciudadano que demanda la sociedad actual.

\subsection{Costa Rica}

Anteriormente, se presentó el abordaje del tema en Chile y Colombia, y como en ambos casos se ha detallado sobre la importancia que tiene la convergencia entre la educación geográfica y la formación 
ciudadana en cada uno de esos países, a su vez se han expuesto pequeños ejemplos de cómo se trabaja este tema en el currículo escolar y los tropiezos que eventualmente tienden a presentarse.

Para el caso particular de Costa Rica, es posible trabajar esta vinculación desde el currículo del Bachillerato en la Enseñanza de los Estudios Sociales y Educación Cívica de la Escuela de Historia de la Universidad Nacional. Dentro de la malla curricular de esta carrera académica, se encuentra el curso denominado Geografía ambiental y riesgo natural, que abarca temas que son de interés para la sociedad actual. En el que se reconoce la importancia que debe existir entre la educación geográfica y la formación ciudadana, y esta vinculación se manifiesta dentro de los objetivos del curso que pretende que el estudiante desarrolle habilidades de ambas disciplinas y que pueda transmitir dichos conocimientos como futuro educador.

El objetivo general de este curso tiene como meta que el estudiante logre "analizar y discutir los elementos conceptuales y prácticos de la geografía ambiental y los riesgos de origen natural como mecanismo para promover una adecuada comprensión del medio y una sociedad participe del desarrollo sustentable". (Avendaño, 2014, p. 1) El curso incentiva al estudiante a reconocer los problemas del entorno en el que habitan, y así promover su participación dentro de la gestión ambiental y de riesgo, buscando el desarrollo sustentable. Este es un ejemplo en el que se evidencia cómo es posible complementar el enfoque geográfico con la participación ciudadana, y así formar ciudadanos con conciencia territorial.

\section{Conclusiones}

La revisión bibliográfica recopilada en este artículo permite discernir lo que se entiende por educación geográfica y formación ciudadana desde la concepción de diversos autores. La educación geográfica dota al individuo de habilidades que le permitan socializar y resolver problemas en el territorio que habita, así como de comprender la realidad que vive y ser consciente del territorio que ocupa, activa su capacidad de análisis espacial y mejora su comprensión para contextualizar diferentes escenarios en un territorio determinado. 
La formación ciudadana capacita al individuo, futuro ciudadano, para la resolución de conflictos en la sociedad en la que convive, lo hace estar consciente del rol activo, participativo y democrático que debe desempeñar, lo incentiva al desarrollo de diversas actividades y promueve su evolución como ciudadano.

Ambas disciplinas están enfocadas en formar ciudadanos que resuelvan conflictos, que se desarrollen y se transformen conforme van adquiriendo habilidades que tengan capacidad de análisis y participen activamente en la sociedad, y además desarrollen estos aspectos en un territorio determinado, limitado y a diversas escalas, que dominen su entorno y sean capaces de reconocerlo y actuar sobre él. Es por ello, que tanto la educación geográfica como la formación ciudadana deben ser integrales, se deben enseñar con una visión holística que permita concebir "ciudadanos territoriales" que dominen el conocimiento brindado por ambas formaciones y que sean "ciudadanos geográficamente alfabetizados."

\section{Referencias}

Araya, F. (2014). Relevancia de la Educación Geográfica en Chile. Chile: Laboratorio de Planificación Territorial, Universidad Católica de Temuco.

Araya, F., Arenas, A., Bruno, C., Figueroa, R., Salinas, V., \& Canihuante, G. (2013). Formación Ciudadana desde la Eduación Geográfica. La Serena: Universidad de La Serena.

Arenas, A., y Salinas, V. (2013). Giros en la Educación Geográfica: renovación de lo geográfico y lo educativo. Revista de Geografía Norte Grande. 56: 143-162

Avendaño, D. (2014). Programa de curso: Geografía Ambiental y Riesgos Naturales. Escuela de Historia, Universidad Nacional. Heredia, Costa Rica.

Buitrago, O.La educación geográfica para un mundo en constante cambio. Obtenido de Geo Crítica: http://www.ub.edu/geocrit/b3w-561.htm

Caro, A. (2012). El aporte de la eduación geográfica a la formacipon ciudadana. Obtenido de Cybertesis: http://cybertesis.ubiobio.cl/tesis/2012/caro_a/doc/caro_a.pdf

Claudino, S., \& Spinelli, F. (2012). Educación geográfica y ciudadanía: un abordaje reflexivo. Obtenido de

EUROGEOGRAPHY: http://www.eurogeography.eu/digitalearth/2012_Educacion_Digital.pdf 
De la Calle, M. (2013). La enseñanza de la geografía ante los nuevos desafíos ambientales, sociales y territoriales. Obtenido de http://www.eurogeography.eu/digitalearth/2012_Educacion_Digital.pdf

EUROGEOGRAPHY:

Flores, M. P. (2012). La educación de género en contextos creativos para un aprendizaje vivencial de ciudadanía. Obtenido de http://es.slideshare.net/ZeebaXtian/educar-para-la-participacinciudadana-en-la-enseanza-de-las-ciencias-sociales-volumen-ii

Gomariz, F., Gómez, C., Miralles, P., \& Ortuño, J. (2012). Ciudadanía y participación en la enseñanza de las ciencias sociales. Una experiencia didáctica a través de la lectura y el análisis de "Rebelión en la granja" de G. Orwell. Obtenido de http://es.slideshare.net/ZeebaXtian/educarpara-la-participacin-ciudadana-en-la-enseanza-de-las-ciencias-sociales-volumen-ii

Gutiérrez, A. (2010). El ciudadano territorial: propósito de la formación ciudadana. Obtenido de UNIPLURI/VERSIDAD, Universidad de Antioquia: http://aprendeenlinea.udea.edu.co/revistas/index.php/unip/article/view/9578/8818

Gutiérrez, A., \& Pulgarín, M. R. (2009). Formación ciudadana: ¡utopía posible! Educación y Pedagogía, 33-48.

Medir, R., \& Magin, C. (2012). Educación para la participación ciudadana en la educación no formal: aportaciones desde el ocio y el tiempo libre. Obtenido de http://es.slideshare.net/ZeebaXtian/educar-para-la-participacin-ciudadana-en-la-enseanza-de-lasciencias-sociales-volumen-ii

Pulgarín, M. (2011). Los estudios del Territorio y su intencionalidad pedagógica. Revista Geográfica de América Central, 1-14.

Pulgarín, M. R. (2011). El espacio geográfico como objeto de enseñanza en el área de las ciencias sociales. Obtenido de Sociedad Geográfica de Colombia: http://www.sogeocol.edu.co/poraut.htm\#P

Rodríguez, F. (2012). Territorio e identidad: Educación geográfica para la construcción de identidades. ANEKUMENE, 10-27.

Souto, X. (2004). La geografía escolar en el período 1990-2003. Obtenido de http://www.uclm.es/seminarios/sehisp/archivos_master/souto/Legislaci\%C3\%B3nGeografia.pdf

Souto, X. (2011). Una educación geográfica para el siglo XXI: Aprender competencias para ser ciudadano en el mundo global.ANEKUMENE, 10-47

\section{Nota de la Autora}

*Académica Interina de la Universidad Nacional. Heredia, Costa Rica. Licenciada en Ciencias Geográficas con Énfasis en Ordenamiento Territorial. E-mail: avahe08@gmail.com 